\title{
BOOK-TAX CONFORMIDADE E GERENCIAMENTO DE RESULTADOS NO BRASIL
}

\author{
BOOK-TAX CONFORMITY AND EARNINGS MANAGEMENT IN BRASIL
}

Antônio Lopo Martinez

University of Coimbra - Portugal

antoniolopomartinez@gmail.com

Renan Muciaccia Almeida

Fucape Business School - Brazil

renan.muciacciaa@gmail.com

\author{
Submissão: 10/1//2018. Aceitação: 01/12/2019. \\ Publicação: 30/12/2019. Sistema de avaliação: Double \\ blind review. Universidade FUMEC / FACE, Belo Horizon- \\ te - MG, Brasil. Editores Prof. Dr. Mário Teixeira Reis Neto \\ - Prof. Dr. Cid Gonçalves Filho.
}

\section{RESUMO}

O objetivo do trabalho foi evidenciar os efeitos da conformidade sobre o gerenciamento de resultados através de metodologias de pesquisas empíricas, no período de 1995 a 2016. Ao todo foram estudadas 280 empresas por ano, utilizadas como amostra do estudo, sendo essas empresas todas listadas na B3 - Brasil, Bolsa, Balcão. A conformidade dos lucros foi analisada por duas metodologias, propostas por trabalhos anteriores, que buscam evidenciar o nível de conformidade e em adicional o nível de diferença entre os lucros. Para análise do gerenciamento foi utilizado o modelo de Jones Modificado, que investiga o comportamento oportunístico dos gestores de manipular os resultados financeiros das empresas. Os resultados encontrados suportam a hipótese estudada, sendo que a conformidade dos lucros gerou efeito sobre o gerenciamento de resultado. Em adicional foram encontrados resultados em que a alavancagem financeira, tamanho da empresa e vendas, podem ter impacto sobre a oportunidade do comportamento dos gestores de gerenciar os resultados.

\section{PALAVRAS-CHAVE}

Book-Tax differences. Book-Tax conformity. Conformidade dos lucros. Gerenciamento de resultados. Comportamento oportunístico. 


\section{ABSTRACT}

The objective of the study was to highlight the effects of conformity on earnings management through empirical research methodologies, from the period 1995 to 2016. In all, 280 companies were studied per year, used as sample of the study, being these companies all listed in B3 - Brazil, Bolsa, Balcão. The conformity of earnings was analyzed by two methodologies, proposed by previous studies, that seek to demonstrate the level of conformity and in addition the level of difference between books. For the analysis of the management was used the Modified Jones model, which investigates the opportunistic behavior of the managers to manipulate the financial results of the companies. The results found, support the hypothesis studied, being that the conformity of the books have effect on the earnings management. In additional was found results that financial leverage, company size and sales, can impact on the opportunity of managers behavior to manage the results.

\section{KEYWORDS}

Book-Tax differences. Book-Tax conformity. Earnings conformity. Earnings management. Opportunistic behavior.

\section{INTRODUÇÃO}

A divulgação das informações contábeis tem como finalidade oferecer, simetricamente, informações necessárias aos investidores, gerentes e outros que se valem delas para tomada de decisões (DECHOW, GE e SCHRAND, 20I0). Para Dechow, Kothari e Watts (1998), o lucro é a informação central da contabilidade; resumidamente, mede a performance de uma empresa. Essas informações apresentadas não estão livres da influência dos controladores das empresas, que, por seus objetivos e incentivos podem de alguma maneira gerenciar essas informações apresentadas (DECHOW, GE e SCHRAND, 20I0).

A qualidade das informações contábeis é influenciada por diversos fatores, sendo altamente demandadas em negociações contratuais (crédito, investimentos, seguros, entre outros) e por incentivos e/ou oportunidades de gerenciamento destes relatórios (valor das ações, dividendos, metas, entre outros) (GIVOLY, HAYN e KATZ, 2010). Tanto para o propósito contratual como para incentivos e oportunidades de gerenciamento, a qualidade dos relatórios contábeis pode sofrer a influência de diversos tipos, em que a conformidade entre os relatórios tributários e societários poderão ter certa influência no resultado da qualidade dos relatórios (GIVOLY, HAYN e KATZ, 2010)

Por um lado, Formigoni et al. (2009) compreende também que os relatórios contábeis além de ser um meio de comunicação da administração sobre a performance da empresa, podem ter um objetivo adicional que é a de auxiliar na apuração dos tributos devidos pela empresa. Dessa forma o Governo pode estabelecer sistemas diferentes para a contabilização e tributação. 
Com essa diferenciação dos sistemas contabilidade financeira e tributária cria-se, também, duas fontes diferentes de resultados (financeiro e tributário), que podem ser diferenças de resultados permanentes ou temporárias (FORMIGONI et al., 2009).

As diferenças de resultado permanente ocorrem quando receitas ou despesas são reconhecidas, mas não possuem efeitos tributários, ao passo que as diferenças temporárias são as diferenças quanto ao momento de reconhecimento de receitas ou despesas. Esses desalinhamentos entre as normas contábeis e tributárias, geram a BTD (book-tax-differences).

Por outro lado, Tang (2005), apresenta duas vertentes para explicar o surgimento da BTD. A primeira vertente defende que a diferença entre os relatórios surge da incompatibilidade das normas contábeis com as normas tributárias. Já a segunda vertente apresenta as escolhas oportunísticas como sendo a causadora do surgimento da BTD, uma vez que os administradores e gestores podem agir de forma discricionária sobre os números contábeis para atender interesses de partes relacionadas.

Apresentadas essas duas linhas de análise do surgimento da BTD por Tang (2005), começa a se entender a relação entre gerenciamento de resultado e a conformidade dos lucros nas empresas. Como no Brasil as normas contábeis sofrem fortes influencias das normas tributárias, verifica-se a importância de se estudar como a tributação pode afetar a qualidade dos relatórios contábeis nas empresas brasileiras e como a conformidade dos lucros pode influenciar as oportunidades dos gerentes de gerenciar ou não as demonstrações contábeis e tributárias das empresas.

Diante dessa possível relação da influên- cia tributária nos relatórios contábeis e das diferentes formas de composição societárias, criou-se a questão da pesquisa deste trabalho, que é: há relação entre o nível conformidade dos relatórios contábeis e tributários das empresas brasileiras com o gerenciamento do resultado?

A evidenciação dentro dos resultados contábeis das empresas de capital aberto listadas na bolsa de valores, se há ocorrência ou não de práticas que podem resultar na melhora ou piora da qualidade das demonstrações, através de práticas discricionárias de gerenciamento de resultados e se há, através dessas práticas de gerenciamento, redução ou aumento na taxa efetiva de recolhimento de impostos.

O escopo do estudo, abrange todas as empresas listadas na bolsa de valores (B3), que não sejam empresas financeiras e/ou seguradoras. $O$ período estudado por teste trabalho será de 1995 a 2016, período este que foi definido por abranger o período antes da adoção do IFRS e após a adoção das regras contábeis do IFRS.

$\mathrm{O}$ estudo tem por finalidade ampliar os entendimentos anteriores, principalmente quando trazidos para o Brasil, sobre os quais ainda se tem poucos avanços nos estudos de qualidade do lucro e gerenciamento de resultados. Trabalhos anteriores, como os de Beatty et al. (2002), Ball e Shivakumar (2005), Givoly, Hayn e Katz (2010), Dechow e Dichev (2002), buscaram evidenciar o gerenciamento de resultados e qualidade de lucro em países como Reino Unido e EUA. No Brasil temos estudos nesse sentido de gerenciamento de lucro como os de Martinez (2006), Coelho e Lima (2007), Formigoni, Antunes e Paulo (2009) Martinez (20l0), Ardison, Martines, Galdi (20l3), Edilson Paulo (2007 - tese 
de doutorado) e Coelho, Galdi e Lopes (20I7). Estudos como o de Tang (20I5), Blaylock et al. (20I7), Atwood et al (2010), Watrin et al. (20l4), Hanlon et al (2008), Hanlon e Shevlin (2005), Sundvick (20I7), entre outros, os quais têm por objetivo encontrar se o nível de conformidade contábil e tributária está relacionada ao gerenciamento de resultado e a qualidade do lucro das empresas. Poucos estudos são direcionados para análise da possibilidade de discricionariedade dos gerentes, que essas métricas apontam e buscam evidenciar esse comportamento oportunístico. Nesse trabalho o foco não será o gerenciamento de resultados em si, mas sim, como essa conformidade entre a contabilidade societária e tributária podem influenciar na qualidade do lucro das empresas.

O estudo contribui para a literatura de forma que ainda não se tem muitos estudos que utilize a conformidade dos lucros como um indicador de possível gerenciamento de resultados nas empresas. Muitos trabalhos focam no BTC (book-tax conformity) como um indicador de gerenciamento de resultados, não analisando que essa conformidade pode explicar o comportamento discricionário, por ela causado, os gerentes para gerenciar resultados, impactando diretamente na qualidade do lucro. Ainda que já exista alguns trabalhos nacionais que estudam o cenário de qualidade do lucro, gerenciamento de resultados, conservadorismo, entre outros, ainda são poucos os avanços que se têm no estudo que a conformidade do lucro pode ter sobre esses resultados.

Importantes estudos como os de Avelar e Santos (20I I), Rosa et al. (20 I0), Machado Benetti e Bezerra (20II), Machado e Beuren (20I4) e Martinez (20I3), fizeram importantes revisões na literatura brasileira sobre o assunto de gerenciamento de resultados, apontando também até onde esses estudos avançaram sobre 0 tema. Importante lembrar, assim como para Martinez (2010), que os estudos empíricos sobre o tema têm grande importância e trazem uma enorme contribuição para os estudos científicos no Brasil, são poucos os avanços em metodologias que não sejam ligadas a esses estudos. Ainda são poucos os estudos de casos e também pesquisas qualitativas, que são importantes ao passo que esses tipos de pesquisas podem apresentar uma análise crítica e filosófica dos resultados (Martins, 20I2).

Este trabalho está organizado na seguinte forma: o primeiro capítulo conta com a introdução, contendo a justificativa, o objetivo e as contribuições do trabalho para a literatura. No capítulo 2 temos o referencial teórico, que apresentará o conceito de Conformidade dos Lucros - Book-Tax Conformity, os conceitos do Book-Tax DIfferences e os conceitos de Gerenciamento de Resultados. No capítulo 3 será apresentada a metodologia do trabalho, na qual se descreve acerca dos dados utilizados, o período estudado e a metodologia aplicada nas análises. No capítulo 4 será apresentado os resultados e as análises dos resultados e, finalmente, no capítulo 5 as conclusões do trabalho.

\section{REFERENCIALTEÓRICO}

Para Coelho, Galdi e Lopes (2017), é menos provável de existir demanda de alta qualidade nos relatórios financeiros das empresas de países emergentes. E que, ainda, em mercados em desenvolvimento os incentivos enfrentados pelas empresas públicas e privadas em gerenciar resultados não tem diferença significante (COELHO, GAL- 
DI e LOPES, 2017). Neste estudo abordaremos as possíveis divergências na qualidade do lucro em períodos com regras contábeis diferentes. Para Tang (20I5), alta conformidade é ligada de forma direta a baixos níveis de gerenciamento e de evasão fiscal, ainda mesmo quando da adoção pelo IFRS, ou controlada por fatores como proteção legal dos investidores, sistema jurídico, desenvolvimento do mercado de capitais.

Este estudo captura o comportamento oportunístico dos gerentes sob a sua discricionariedade nas divulgações contábeis, - que reforça os resultados, pois permite evidenciar que a alta ou baixa conformidade pode deter o gerenciamento de resultados e evasão tributária.

\section{Conformidade dos lucros - Book-Tax Conformity}

Para Sundvik (20I7), a conformidade dos lucros, em geral, é a associação entre a receita financeira e a receita tributável, em que um alto nível de conformidade é ligação a um único sistema para os propósitos de contabilidade e tributação, com a finalidade de usar diretamente a contabilidade financeira para calcular a receita tributável.

Conforme os trabalhos internacionais, duas linhas de debate se firmaram, proponentes e os oponentes da conformidade dos lucros. Proponentes da conformidade dos lucros justificam seu posicionamento com base no aumento da conformidade, pois esse aumento pode levar a restrição da evasão fiscal e diferenças nos lucros contábeis e fiscal, o qual, segundo a linha de posicionamento dos proponentes, diminui a transparência nas demonstrações contábeis e diminui a simetria informacional entre os gerentes e investidores (TANG, 2015; DESAI, 2005;WHITAKER 2005; YIN, 200I).
Segundo Desai (2005), a discricionariedade proporciona a oportunidade de manipular os resultados, aumentando a conformidade limitará as ações dos gerentes de usar essa discrição em favor pessoal. Para Whitaker (2005), os custos para publicar altos ganhos para os investidores e baixo lucro para tributação, pode limitar esse comportamento oportunístico dos gerentes, que podem levar a penalidades que a empresa não estaria disposta a se sujeitar. Whitaker (2005) ainda sugere que baixa conformidade dos lucros contábil e fiscal podem facilitar a ação dos gerentes de inflar os ganhos e reduzir os tributos, no mesmo período.

Já os oponentes do aumento da conformidade (ALI E HWANG, 2000; HANLON et al., 2005; HANLON E SHEVLIN, 2005; ATWOOD et al., 20I0), têm como suporte a restrição da flexibilidade das divulgações contábeis, que podem também levar a um aumento da intervenção de forças políticas, interferências governamentais, nas demonstrações contábeis. Para Atwood (2010), as demonstrações contábeis têm como objetivo reportar as informações financeiras, a posição econômica e de performance da empresa, que não são de interesses tributários. Dessa forma, alta conformidade pode levar os gerentes a se reportarem com mais preocupação em minimizar os efeitos tributários do que na transparência dos resultados financeiros das empresas. Por fim, alta conformidade resulta na perda de informações que seriam importantes para o mercado de capital (TANG, 20I5).

Em meio a esse debate, alguns estudos indicam que não há efeito significante da conformidade ou não dos lucros contábil e fiscal sobre o gerenciamento de resultados. Estudos como os de Burgstahler et al. (2006), 
Watrin (20I2) e Blaylock et al. (20I I), não encontram evidências para suportar a proposição ou oposição à conformidade. Burgstahler et al. (2006), encontrou que a resposta à conformidade dos lucros é diferente para empresas públicas e empresas privadas.

Já Watrin (20I2), apesar de apontar que empresas com alta conformidade nas demonstrações contábeis e tributárias têm um nível de gerenciamento de resultados maior, não concluíram se a conformidade está diretamente ligada ao gerenciamento de resultados. Por sua vez, Blaylock et al. (20I2), encontram que empresas com alta conformidade estão mais engajadas ao gerenciamento, estando os resultados associados à suavização dos lucros, levando em consideração os efeitos tributários e os riscos do gerenciamento.

Esses estudos consideraram modelos diversos de estudos, que não estão focados diretamente na relação da conformidade dos lucros com o gerenciamento de resultados. Alguns estudam os efeitos das diferenças permanentes entre os lucros, outros pesquisam o comportamento das empresas no tempo e em relação à conformidade.Alguns trabalhos estão focados em empresas dos EUA, outros em empresas localizadas em países europeus e outros estudam diversos países em diversos continentes.

A literatura nacional não vem adotando as análises da conformidade sobre o efeito no gerenciamento. Muitos trabalhos adotam as métricas de conformidade como uma proxy de gerenciamento, porém não analisam seus efeitos sobre o gerenciamento.

\section{Gerenciamento de resultados}

Haely eWahlen (1999), descrevem como sendo os relatórios financeiros um meio de transmitir a informação dos gestores sobre a sua performance financeira na empresa, permitindo que gestores possam exercer sua influência sobre os mesmos. As auditorias não são totalmente perfeitas, assim gerentes podem utilizar do seu conhecimento sobre a empresa e seus negócios, para apresentar, da sua forma, métodos, estimações e divulgações que vão ao encontro com as necessidades da empresa, podendo potencializar o seu valor contábil (HAELY e WAHLEN, ( 999). Por fim, gerenciamento de resultados é a prática dos gestores de decidir por métodos, estimações e divulgações que podem não corresponder com a realidade da empresa.

O estudo de Dechow et al. (1996), indica que uma importante motivação para o gerenciamento de resultados das empresas é o desejo de atrair recursos financeiros externos a baixo custo. $O$ mesmo estudo também evidenciou que empresas que fazem o gerenciamento de resultados são menos auditadas, tem mais chances de ter o fundador da empresa como presidente, que também é o presidente do conselho dos acionistas e possivelmente não tem um grande grupo de acionistas que fiscaliza e monitora as decisões tomadas nas empresas.

Para Givoly et al. (20I0), empresas públicas podem ter incentivos ao gerenciamento de resultados, pois, estão sobre forte pressão dos investidores, para que os resultados da empresa seja o mesmo que o esperado pelos analistas. Outros motivos para o gerenciamento de resultados é que os gerentes das empresas estão sob contínua pressão dos investidores para que os resultados das empresas sejam aqueles previstos pelos analistas, seja evitando reportar perdas ou redução do lucro. Ainda para Givoly et al. (2010), empresas públicas 
possuem incentivos para não praticarem gerenciamento nos seus resultados, seja no sentido de evitar processos judiciais e também para reduzir o seu custo de capital (aumentando a sua transparência).

\section{Book-Tax Differences (BTD)}

Ainda existem propósitos divergentes na divulgação das informações contábeis, sendo que na regulamentação desempenhada pelo Estado, as informações contábeis são para o auxílio na determinação do valor do imposto a ser arrecadado aos cofres públicos pela empresa (SUNDER, 1997). Por outro lado, temos que, a regulamentação contábil estabelece um conjunto de normas e procedimentos que auxiliam as empresas na divulgação de suas demonstrações contábeis afim de prestar informações aos usuários externos (FORMIGONI, ANTUNES e PAULO, 2009).

Esses dois tipos de relatórios contábeis para fim de atender as divergentes regulamentações existentes, contábil e tributária, resultam em valores diferentes, que são chamados de Book-Tax-Differences (BTD).

Para Tang (2006), as diferenças entre os resultados contábeis e os resultados fiscais, podem ser analisados por duas linhas de estudos, onde: (i) a primeira linha visa o estudo sobre as diferenças institucionais, ponderando que as diferenças entre relatórios são resultantes, também, das diferenças entre as normas contábeis e as normas tributárias e, (ii) a segunda linha visa o foco dos estudos nos incentivos e escolhas oportunísticas, apontando que os julgamentos sobre os números contábeis e/ou resultados tributáveis, são influenciados de forma divergentes pelos administradores afim de atender os interesses dos gerentes.

Entrando no mérito da discussão do
BTD (Book Tax Differences), como foi apresentado por Tang (2006), as linhas de estudo sobre o assunto estão diretamente relacionadas a qualidade do lucro das empresas, uma vez que os incentivos e oportunidades se confundem e impactam de forma direta nos resultados fiscais e contábeis, assim como na divulgação das informações da empresa.

O trabalho de Ramos Ferreira et al. (20I2) conclui que, o nível de gerenciamento de resultados, apurado através da evidenciação do conservadorismo (accruals) das empresas, e o montante da BTD são diretamente proporcionais, ou seja, "quanto maior o valor da BTD em termos absolutos, maior o valor dos accruals discricionários" (RAMOS FERREIRA et al., 2012). Ainda sobre os achados de Ramos Ferreira et al. (20I2) no estudo das firmas brasileiras, foram encontradas evidências que o tamanho da firma está inversamente relacionado aos acúmulos (accruals) discricionários e a BTD, ou seja, "quanto maior o tamanho da firma menor o nível de práticas discricionárias sobre o lucro da firma".

$A$ intenção deste estudo em verificar a ocorrência da BTD (Book Tax Differences) nos tipos de empresas que são objetos deste trabalho é de poder confrontar se, BTC e BTD podem levar a essa diferença apresentada através de gerenciamento de resultados.

Considerando as características locais, observa-se que a legislação tributária brasileira tem forte influência nas normas contábeis (LOPES e MARTINS, 2007), fazendo com que o estudo da qualidade dos números contábeis e a observância ou não do gerenciamento de resultados - tendo as empresas os incentivos diversos que os 
dois tipos de agressividade fiscal estudadas neste trabalho podem ter - geram uma pior ou melhor qualidade do lucro.

\section{Hipótese}

Considerando as proposições dos oponentes e proponentes da conformidade dos lucros, onde resumidamente, os oponentes pontuam que a conformidade dos lucros pode gerar a perda de informações relevantes para o mercado de capitais - devido a utilização diversa do fisco em relação ao mercado dessas informações - e também o aumento da interferência governamental e do fisco nas divulgações dos relatórios financeiros das empresas. Os proponentes, por sua vez, apoiam o aumento da conformidade pois, com o aumento da conformidade dos lucros contábeis e fiscais, haverá uma diminuição das oportunidades dos gerentes de exercer qualquer influência nos relatórios financeiros das empresas, seja qual for o motivo. Haverá também, segundo os proponentes, uma diminuição da evasão fiscal, pois o fisco terá mais acesso aos números contábeis das empresas e facilitará qualquer processo de fiscalização sobre elas.

Estudos internacionais que estão avançando nos debates e achados sobre a proposição ou oposição da conformidade dos lucros, divergem sobre a ponderação de que o aumento ou a diminuição da conformidade dos lucros podem trazer benefícios ou malefícios aos resultados contábeis e qualidade informacionais. Esse estudo avança também sobre o campo político dos países que estão passando por um momento de transição no seu sistema contábil ou tributário, uma vez que essa proposição ou oposição da conformidade dos lucros podem impactar diretamente sobre as políticas públicas e sobre a arrecadação tributária. Por outro lado, o avanço da adoção ou não de sistemas de baixa ou alta conformidade do lucro podem levar a um cenário adverso de investimentos nas empresas, uma vez que a conformidade do lucro implica diretamente sobre a qualidade dos resultados contábeis e sua utilização informacional.

No quadro I a seguir apresentamos os achados de trabalhos que estudaram a conformidade dos lucros, sua relação com o gerenciamento de resultados, suas implicações na qualidade da informação contábil e implicações fiscais sobre o tema.

Diante da construção apresentada anteriormente, considerando as particularidades do BTC, do BTD e suas influências sobre o gerenciamento de resultados, apresenta-se a seguinte hipótese de estudo:

H: Há relação entre a conformidade dos lucros contábil e fiscal na diminuição do gerenciamento de resultados.

\section{METODOLOGIA DE PESQUISA}

Os modelos e a metodologia aplicada neste trabalho para testar a hipótese, foram adaptados dos trabalhos de Atwood et al. (20I0) e Tang (20I5), que realizaram investigações acerca do BTC, BTD e Gerenciamento de Resultados. Este estudo foi realizado na forma de uma pesquisa empírica com o uso de técnicas de análise estatística multivariada. Os modelos a serem implementados foram testados a partir da ferramenta de Análise de Regressão Múltipla com o auxílio do software STATA.

\section{Base de dados}

Os dados analisados serão colhidos e será constituída uma amostra das companhias abertas brasileiras listadas na B3 Brasil, Bolsas, Balcão. A amostra é compos- 


\section{QUADRO 1 - resultaods e achados internacionais}

\begin{tabular}{|l|l|}
\hline Sundvik (2017) & $\begin{array}{l}\text { Os achados suportam a ideia de que a alta conformidade está associada a } \\
\text { baixo nível de gerenciamento de resultados e num cenário de fortes incentivos } \\
\text { tributários, a conformidade está associada ao gerenciamento dos lucros para } \\
\text { baixo. Por fim, indica que há benefícios tributários com o gerenciamento com } \\
\text { a baixa conformidade. }\end{array}$ \\
\hline tang (2015) & $\begin{array}{l}\text { Os resultados indicam que a alta conformidade reduz o gerenciamento de re- } \\
\text { sultados e evasão fiscal, assim, suportando o posicionamento de proposição } \\
\text { do aumento da conformidade dos lucros. }\end{array}$ \\
\hline blaylock et al. (2015) & $\begin{array}{l}\text { Altos níveis de conformidade não estão necessariamente ligados a menos } \\
\text { gerenciamento de resultados. Os achados indicam o contrário, ou seja, que } \\
\text { altos níveis de conformidade estão ligados a altos níveis de gerenciamento. } \\
\text { No entanto, não é conclusivo que a baixa conformidade está associada a } \\
\text { baixos níveis de gerenciamento. }\end{array}$ \\
\hline watrin et al. (2014) & $\begin{array}{l}\text { Resultados indicam que alto nível de conformidade pode reduzir o gerencia- } \\
\text { mento de resultados. Os lucros são menos gerenciados em empresas que } \\
\text { adotam o sistema único de demonstração do que em empresa que adotam o } \\
\text { sistema com dois tipos de demonstração. }\end{array}$ \\
\hline Atwood et al. (2010) & $\begin{array}{l}\text { Aumentar a conformidade dos lucros pode resultar em reportar lucros me- } \\
\text { nos consistentes. Os resultados sugerem que migrar para um cenário de alta } \\
\text { conformidade pode ocasionar na piora da qualidade informacional dos lucros, } \\
\text { indicando um aumento no gerenciamento de resultados. }\end{array}$ \\
\hline
\end{tabular}

Fonte: Autores.

ta por empresas selecionadas conforme o enquadramento do segmento de atividade, como listado pela própria B3. Foram excluídas da base, as empresas do segmento financeiro e de seguros, por terem significantes diferenças no modelo de demonstração contábil e tributário utilizados pelas empresas desses segmentos.

Este trabalho é feito com dados do período de 1995 a 2016, nos quais podemos observar nesse período a mudança do modelo de demonstração contábil no país (adoção do IFRS). A opção por esse período de estudo é um fator essencial para os resultados que é proposto nesse estudo, uma vez que havendo uma mudança no cenário contábil e fiscal podemos analisar como esse impacto pode ter efeito nas decisões dos gerentes das empresas, quanto ao gerenciamento de resultado.

Tendo em vista que no período anterior à adoção do IFRS, o modelo de demons- tração contábil e fiscal eram muito próximos, sofrendo forte influência do governo e após a adoção dos padrões das regras internacionais de contabilidade, limitou-se muito esse cenário de influência governamental e do fisco sobre a divulgação dos resultados contábeis. $O$ estudo se limita ao ano de 2016, pois este é o período mais recente das divulgações contábeis necessárias ao estudo.

Esses dados serão colhidos nas bases do programa Economática. Serão analisadas ao todo uma base de 280 empresas, que pelo período que serão analisadas totalizará em 5.600 observações.

\section{Modelos de pesquisa}

A metodologia utilizada para testar a hipótese foi adaptada dos trabalhos de Atwood, Drake e Myers (20I0) e Tang (20I5), que realizaram investigações semelhantes. Este estudo trata-se de pesquisa empírica com 
TABELA 1 - Composição da amostra

\begin{tabular}{lc}
\hline Descrição & Observações \\
\hline Empresas brasileiras de capital aberto ativas na B3 & 368 \\
\hline (-) Exclusão de empresas do setor financeiro e seguro & $(56)$ \\
\hline (-) Exclusão de empresas classificadas como ‘OUTROS’ & $(32)$ \\
\hline (=) Quantidade de empresas investigadas & 280 \\
\hline (x) Quantidade de anos investigados & 20 \\
\hline (=) Quantidade de observações (empresa/ano) & 5.600 \\
\hline
\end{tabular}

Fonte: Elaborado pelo autor.

o uso de técnicas de análise estatística multivariada com o auxílio do software STATA. Os modelos a serem implementados foram testados a partir da ferramenta de Análise de Regressão Múltipla.

\section{Medidas de conformidade do book-tax mandatórias.}

Pesquisas anteriores investigaram o problema de book-tax conformity empregando um indicador variável para classificar um país como exibidor de alta ou baixa conformidade, baseados em julgamentos subjetivos identificando vários preceitos legais nas regras contábeis e fiscal (Ali e Hwang, 2000; Hung, 200I). Essa abordagem, no entanto, é ineficaz para quantificar o nível de conformidade através de países e suas mudanças no tempo.

Atwood et al. (2010) desenvolveu uma medida contínua de conformidade usando o ranking do RMSEs, estimado de um modelo de regressão da despesa corrente de impostos (currente tax expenses - CTE) no lucro antes do imposto (pre-tax book income), outros impostos e dividendos. Os autores assumem que o lucro antes do imposto explica a despesa corrente com impostos quando o relatório contábil e o fiscal estão altamente alinhados, a porção não explicada pelo modelo reflete a extensão que os gerentes possuem de relatar lucros diferentes do lucro tributável.Atwood
(2010) mede a conformidade mandatória através do modelo a seguir:

CTE_it $=\beta \_0+\beta \_1$ PTBI it $+\beta \_2$ DIV it $+\varepsilon_{-}$it

Em que, (current tax expense) é despesa corrente com impostos, t é o indicador de ano, (pre tax book income) é o lucro antes do imposto (LAIR), é o total de dividendos, e é o termo de erro.Todas as variáveis são escalonadas pela média total de ativos. Foi nomeado o RMSE estimado do modelo I, como RMSEadm. No trabalho de Atwood et al. (20I0) foi ranqueado o RMSE do modelo I por país-ano em ordem decrescente (o maior RMSE num ano é ranqueado 0 e o mais baixo naquele é $n-I$, onde $n$ é o número de empresas incluídas naquele ano) e então dividiu por $\mathrm{n}$-I para escalonar a medida entre 0 e $\mathrm{I}$.

Como neste estudo não teremos a análise com vários países, como foi a proposta de Atwood et al. (2010) e Tang (20I5), em que o resultado do ranking sinaliza o grau de conformidade, utilizaremos os valores absolutos do RMSE. Essa medida será rotulada como BTC, onde quanto menor o RMSE do BTC maior será o nível de conformidade.

Uma grande preocupação relacionada ao BTC é que o uso oportunístico da discricionaridade dos gerentes pode também levar a um alcance mais amplo da despesa corrente de impostos (CTE) do que a em- 
presa pode reportar para um dado nível de lucro antes do imposto (PTBI). Que conste, empresas engajadas em mais gerenciamento de resultados ou evasão fiscal irão exibir uma variação maior no CTE que firmas que não o fazem, mesmo que essas empresas estejam sujeitas ao mesmo nível de conformidade (ATWOOD et al. 2010;TANG, 20I5). Na comparação cross-country, países com alta atividade de gerenciamento de resultados e evasão fiscal vão produzir um maior RMSE, o que contamina a conformidade mandatória. Para aliviar essa preocupação, Tang (20I5) desenvolveu uma nova medida de conformidade mandatória usando fontes regulatórias do BTD.

BTD por definição, origina-se da diferença legal entre os padrões contábeis e as regras tributárias. Teoricamente, empresas em países com baixa conformidade reportam alta BTD, enquanto empresas em países com conformidade total não vão ter BTD (TANG, 20I5). Dessa forma, para mitigar problemas da análise do BTC, a próxima proxy a ser utilizada será a BTD, por ser uma ótima proxy de conformidade (TANG, 20I5).

Segundo a literatura indica, as manipulações gerenciais no lucro e impostos também aumentam a variação no BTD. Para filtrar esses erros, foi feita a regressão do BTD total no gerenciamento de resultados (medido como accruals discricionários), evasão fiscal (medido pela diferença entre alíquota legal do imposto e alíquota efetiva de imposto), e o termo de interação. O RMSE dessa regressão representa a conformidade mandatória pois captura as diferenças que estão relacionadas à regra. Sendo assim, o resultado de alto RMSE indica baixa conformidade.

$$
\mathrm{BTD}=\beta \_0+\beta \_1 \mathrm{DACC}_{-} \mathrm{i}+\beta_{-} 2 \mathrm{TP} \mathrm{i}
$$
$+\beta \_3$ DACC*TP_i $+\varepsilon_{-} \mathrm{i}$
Em que, BTD é calculada pelo lucro antes do imposto multiplicado pela alíquota legal menos despesas correntes com impostos. DACC são os acrruals discricionários, o resíduo estimado de um modelo cross-secction, Jones modificado com atraso no retorno de ativos por país para cada empresa (KOTHARI et al., 2005). Foram utilizados os accruals discricionários pois eles capturam o uso oportunístico de discricionariedade dos gerentes nas regras contábeis. TP é a medida de evasão fiscal, calculada pela subtração da alíquota legal menos a alíquota efetiva (CETR), que é a proporção de despesa corrente com impostos para fluxo de caixa operacional. Foi utilizado o valor absoluto do RMSE, sendo que, quanto maior o RMSE da BTD, menor será a conformidade. $A$ seção anterior desenvolveu o proxy de conformidade que captura a extensão da conformidade através das empresas num dado ano.

\section{Medida de Gerenciamento de Resultados}

Para consistência, nesta seção, é construído uma escala de gerenciamento de resultados para as empresas brasileiras. Para estimar o nível de gerenciamento através das empresas, será empregado à medida em que tem sido usada em pesquisas anteriores de accruals discricionários.

Desenhado por pesquisas contábeis anteriores (DECHOW et al. 1996; KOTHARI et al., 2005), foram estimados os accruals discricionários usando a seguinte equação:

TACC_it $=\beta \_0+\beta \_1$ (1/AT_it) $+\beta \_2$ $\left(\right.$ REV - AR) it + $\beta \_3$ PPE_it $+\beta_{-} 4$ ROA_it $+\varepsilon_{-}$it

Em que, é o total de accruals, mensurado como a mudança dos ativos correntes mais a mudança nos débitos de curto 
prazo menos a soma do passivo circulante, mudança no caixa e despesas com depreciação e amortizações no ano t. é a mudança nas vendas do ano $\mathrm{t}$ - I para o ano $\mathrm{t}$. REV é a mudança nos recebíveis do ano t-I para o ano t. AR é propriedade plantas e equipamentos, que neste estudo representará o ativo imobilizado, no ano t. todas essas viáveis são escalonadas pelo ativo total (I/AT_it). ROA é o retorno dos ativos, mensurados pelos ganhos divididos pelo ativo total.

A inclusão do termo de intercepto é uma sugestão do trabalho de Kothari et al. (2005) e aplicado também no trabalho de Tang (20I5), que sugerem que este termo ajuda a aliviar a heteroscedasticidade e atenua a preocupação quanto às variáveis omitidas, além de produzir uma medida mais simétrica de accruals discricionários (TANG, 20I5). Accruals discricionários (DACC) são os resíduos estimados do modelo 3 . $O$ alto DACC, indica elevado nível de uso oportunístico de discricionariedade no relatório financeiro, incluindo gerenciamento de resultados para cima ou para baixo. $\bigcirc$ valor do DACC é obtido pelo valor absoluto do RMSE e esse RMSE será utilizado como index de gerenciamento de resultados (EM), que é a média do gerenciamento por ano.

\section{Teste para o impacto da conformidade no gerenciamento de resultado}

Foi conduzido uma análise anual para acessar onde conformidade é associada com gerenciamento de resultados e evasão fiscal por duas razões. Primeiramente, o foco deste estudo foi o de examinar a variação na conformidade, a qual varia em nível dos anos e não em nível das empresas (TANG, 20I5). Em segundo lugar, fatores específicos nacionais e estruturais demons- tram ser relativamente estáveis no mesmo país e período, a análise anual por empresa pode elevar o nível de significância. Foi utilizado o seguinte modelo (4), proposto por Tang (20 I5), para testar a hipótese:

$\mathrm{EM}=\alpha \_1+\alpha \_2 \mathrm{CONF}+\alpha \_3$ dIFR$\mathrm{S}^{*} \mathrm{CONF}+\alpha \_4$ TAM $+\alpha \_5$ VENDAS+ $\alpha \_6$ $\mathrm{ALAV}+\alpha \_7 \mathrm{ROA}+\varepsilon$

Em que, é o gerenciamento de resultados conforme foi apresentado pelo modelo (3), medido pelos erros absolutos obtidos através da regressão proposta, que indicam que quanto maior o erro maior é o gerenciamento na empresa naquele ano. A variável apresenta a conformidade estimada pelas proxys geradas dos modelos I e 2 , que indicam o nível de conformidade das empresas, por meio dos erros absolutos obtidos pelos modelos, de forma decrescente, apontam que, quanto maior o erro menor será a conformidade da empresa naquele ano. A é uma variável criada a partir dos erros calculados pelos modelos I e 2 ( e ), conforme foi aplicado por Tang (20I5) em seu trabalho. A interação dessa variável com a adoção ou não dos modelos internacionais do IFRS é a segunda variável do modelo apresentada por , a qual demonstra o nível de conformidade com a adoção do IFRS.

Foram utilizadas também variáveis de controle para monitorar as especificidades de cada empresa, quais sejam, tamanho, vendas, alavancagem financeira e retornos sobre os ativos. Para Tang (2015) essas variáveis - também foram utilizadas nos trabalhos de Atwood et al. (2010) e Burgstahler et al. (2006) -mostraram-se muito associadas à agressividade fiscal e ao gerenciamento de resultados. $A$ medida da variável tamanho (TAM) é feita pelo logaritmo natural do ativo total da empresa no 
ano. A variável venda (VENDAS) é medida pelo valor total da receita bruta dividido pelo ativo total, naquele ano. Alavancagem financeira ( $A L A V)$ é uma variável calculada pela divisão do passivo total pelo ativo total. Por fim, a variável do retorno sobre os ativos (ROA) é calculada pela divisão do resultado líquido pelo ativo total.

\section{RESULTADOS \\ Modelo I - BTC (Book-Tax Conformity)}

No modelo (I) de Atwood et al. (2010) temos que a variável dependente é assinada por CTE (current tax expenses), que são as despesas correntes com IRPJ e CSLL.As variáveis independentes são assinadas pela PBTI (pre book tax income), que é o lucro antes do imposto (LAIR) e DIV, que são os dividendos pagos.

Com a finalidade de controlar possíveis outliers na amostra, todas as variáveis apresentadas pelo modelo foram divididas pela média do total dos ativos. Também para limpar a amostra obtida foram removidos os possíveis valores negativos que poderiam ter a variável PBTI (LAIR). Na tabela 2 , apresentamos uma estatística descritiva do modelo com os valores de número de amostras, média da variável na amostra, mediana da variável, desvio padrão, valor mínimo e valor máximo da amostra da modelagem, conforme segue a tabela:

Os resultados apresentados na Tabela 3, da regressão do modelo (I) proposto por Atwood et al. (20l0), atestam que o modelo proposto é válido (p_valor $\mathbf{= 0 , 0 0 0}$ ). Valor de explicação encontrado no modelo é de $78,62 \%\left(\mathbf{R}^{2}\right.$ ajustado $\left.=\mathbf{0 , 7 8 6 2}\right)$, o que significa que ele consegue explicar a variável CTE, ao grau de poder de explicação de 78,62\%, pelas variáveis regredidas PTBI e DIV. A diferença de explicação de $21,38 \%$ está associada a outras variáveis não testadas ou a erros aleatórios. Como resultado da regressão nota-se que somente a variável PTBI é significante e tem poder explicativo sobre a variável dependente. Importante observar que na regressão aplicada do modelo, as variáveis explicativas nos permitem dizer que quanto maior o lucro antes do imposto de renda PTBI (LAIR), maior será o custo efetivo dos impostos. $O$ erro do modelo é verificado, de forma decrescente, em que quanto maior o erro, menor será a conformidade daquela empresa naquele ano.

Da regressão do modelo foram extraídos

TABELA 2 - Modelo de BTC - Book-Tax Conformity

\begin{tabular}{l|c|c|c|c|c|c}
\hline & $\mathrm{N}$ & Média & Mediana & Desv. Padrão & Min. & Max. \\
\hline CTE $^{1}$ & 4466 & 0.02 & 0.00 & 0.12 & -3.07 & 2.48 \\
\hline PBTI $^{2}$ & 4418 & 0.05 & 0.00 & 0.44 & -10.56 & 7.84 \\
\hline DIV $^{3}$ & 4500 & 0.01 & 0.00 & 0.07 & 0.00 & 1.51 \\
\hline Erro do modelo (BTC) & 4363 & 0.02 & 0.00 & 0.11 & -2.36 & 1.84 \\
\hline
\end{tabular}

TABELA 3 - Modelo de BTC - Book-Tax Conformity

\begin{tabular}{c|c|c|c|c}
\hline & Coeficiente & Std. Error & T & Signif.(P) \\
\hline PTBI & .2328437 & .024477 & 9.51 & $0.000^{*}$ \\
\hline DIV & .0622252 & .1173395 & 0.53 & 0.596 \\
\hline (Constante) & .003108 & .0009827 & 3.16 & 0.002 \\
\hline
\end{tabular}

N. de observ. = 3766; Significância do modelo = 0,000; $\mathrm{R}^{2}=0.7862 ; \mathrm{RMSE}$ (erro) $=.05647$

*significância a $1 \%$ **significância a $5 \%$ *** significância a $10 \%$ 
os erros (estatística descritiva na Tabela 2), que são os valores de RMSE por empresa/ ano, que serão utilizados em valores absolutos como proxy de conformidade, denominada BTC, para regressão no modelo 4.

\section{Modelo 3 - Jones Modificado (Accruals Discricionários)}

Para medir o gerenciamento de resultado, foi utilizado o modelo de Jones Modificado (DECHOW et al. 1995; KOTHARI et al. 2002), o qual é aplicado na medição dos accruals totais. Essa medida é utilizada para capturar a diferença entre as regras de divulgação das demonstrações e o uso oportunístico da discricionariedade dos gerentes nessas demonstrações (TANG, 20I5).

A Tabela 4 apresenta uma estatística descritiva do modelo contendo na apresentação da estatística, o número de amostras, média da variável na amostra, mediana da variável, desvio padrão, valor mínimo e valor máximo da amostra da modelagem, conforme segue a tabela:
A seguir na Tabela 5, são expostos resultados da regressão do modelo proposto de Jones, modificado pelos trabalhos de Dechow et al. (1995) e Kothari et al. (2005), utilizado por Tang (20I5), no seu estudo sobre conformidade.

De acordo com o resultado da regressão do modelo proposto, identificamos que o modelo é valido ( $p$ valor $=\mathbf{0 , 0 0 0}$ ) e o seu poder explicativo é de $48,03 \%$ $\left(R^{2}\right.$ ajustado $\left.=\mathbf{0 , 4 8 0 3}\right)$, ou seja, pelas variáveis utilizadas conseguimos explicar $48,03 \%$ dos accruals totais. Podemos concluir que, para os accruals totais, todas as variáveis independentes são importantes a um grau de confiabilidade de $99 \%$. Com os resultados é possível concluir que, variáveis não estudadas pelo modelo e outros efeitos aleatórios têm poder de explicação de $5 \mathrm{I}, 97 \%$ da regressão de accruals totais.

Como a intenção do estudo do modelo de Jones Modificado, neste trabalho, é obter os acrruals discricionários, que são obtidos extraindo os erros do modelo, então serão considerados como accruals discricionários

TABELA 4 - Modelo de Jones Modificado - Accruals Discricionários

\begin{tabular}{l|c|c|c|c|c|c}
\hline & $\mathrm{N}$ & Média & Mediana & Desv. Padrão & Min. & Max. \\
\hline TACC $^{5}$ & 4369 & 1.38 & 0.23 & 6.18 & 0.00 & 124.33 \\
\hline TAM & 4421 & 0.00 & 0.00 & 0.00 & 0.00 & 0.33 \\
\hline RECb_REC & 4405 & 0.54 & 0.07 & 2.47 & -0.51 & 51.46 \\
\hline PPE & 3795 & 0.00 & 0.00 & 0.00 & 0,00 & 0.00 \\
\hline ROA & 4416 & 0.70 & 0,59 & 0,56 & $-0,02$ & 5,84 \\
\hline Erro (DA) & 4503 & 0.26 & 0.21 & 0.37 & 0.21 & 3.81 \\
\hline
\end{tabular}

TABELA 5 - Modelo de Jones Modificado - Accruals Discricionários

\begin{tabular}{l|c|c|c|c}
\hline & Coeficiente & Std. Error & $\mathrm{t}$ & Signif.(P) \\
\hline TAM & -10.24426 & 3.340387 & -3.07 & $0,002^{*}$ \\
\hline RECb_REC & 1.535014 & .0877514 & 17.49 & $0,000^{*}$ \\
\hline PPE & -1452.62 & 229.6932 & -6.32 & $0,000^{*}$ \\
\hline ROA & -.3317934 & .0242247 & -13.70 & $0,000^{*}$ \\
\hline (Constante) & .3911035 & .0284606 & 13.74 & 0,000 \\
\hline
\end{tabular}

N. de observações = 2236; Significância do modelo $=0,000 ; R^{2}=0.4803 ;$ RMSE (erro) $=.40714$

*significância a $1 \%{ }^{* *}$ significância a $5 \%{ }^{* * *}$ significância a $10 \%$ 
os resíduos estimados pela regressão do modelo 3. Esses erros compõem a variável DACC usada no modelo 2 e que também será a medida utilizada como gerenciamento de resultado (EM) do modelo 4.

\section{Modelo 2 - BTD (Book-tax differences)}

Em adicional ao modelo I, Tang (20I5) desenvolve uma nova medida para fortalecer os resultados de conformidade dos lucros estudados pelo modelo anterior. Esse modelo irá capturar as diferenças entre o lucro contábil e fiscal (book-tax diferences). Para isso, Tang (20 I5) utiliza como variável dependente a BTD, que é calculada multiplicando o valor do lucro antes do imposto de renda (LAIR) pela alíquota legal (34\%) e em seguida diminuindo o valor da despesa corrente com impostos. A variável BTD é controlada pela média do total dos ativos. Para esse modelo foi criado uma variável de accruals discricionários (DACC), que são os erros estimados pelo modelo de Jones Modificado (Dechow et al. 1995; Kothari et al. 2005). Essa medida captura o poder do uso discricionário dos gerentes. Outra variável do modelo é uma medida de evasão fiscal (TP), calculada pela diferença da alíquota legal (34\%) menos a alíquota efetiva do imposto pago, que é entrada pela divisão da despesa corrente com impostos pelo fluxo de caixa operacional.

Na Tabela 6 é apresentada a estatística descritiva do modelo, com as variáveis do mode- lo, contendo na apresentação da estatística, o número de amostras, média da variável na amostra, mediana da variável, desvio padrão, valor mínimo e valor máximo da amostra da modelagem, conforme segue a tabela:

$\mathrm{Na}$ Tabela 7, apresentamos os resultados da regressão do modelo 2 , criado e utilizado por Tang (20/5) no seu estudo sobre conformidade. Foi incluído ao modelo de Tang (20I5), um dummy da adoção ou não do IFRS, essa dummy assumindo o valor de 0 nos anos de 1995 a 2007 e I nos anos de 2010 a 2016. Os resultados da regressão do modelo indicam que somente $6,5 \%$ $\left(R^{2}\right.$ ajustado $\left.=0,065\right)$ das diferenças nos lucros contábil e fiscal são explicados pelas variáveis utilizadas, ou seja, 93,5\% da diferença entre os lucros é explicada por outras variáveis não estudadas no modelo ou por erros aleatórios.

De acordo com os resultados do modelo, os accruals discricionários é uma variável significante que pode explicar a diferença nos lucros contábil e fiscal, a uma confiabilidade de $99 \%$ e tem impacto positivo sob a diferença dos lucros, ou seja, quanto maior os accruals discricionários, maior será a diferença entre os lucros.

\section{Modelo 4 - impacto da conformidade sobre o gerenciamento de resultado}

O modelo 4, proposto por Tang (20I5), é um modelo de regressão utilizado para testar e identificar o efeito que a confor-

TABELA 6 - Modelo de BTD - Book-Tax Differences

\begin{tabular}{l|c|c|c|c|c|c}
\hline & $\mathbf{N}$ & Média & Mediana & Desv. Padrão & Min. & Max. \\
\hline BTD & 4417 & 0.00 & 0.00 & 0.07 & -1.65 & 1.10 \\
\hline DACC & 3503 & 0.26 & 0.21 & 0.37 & -3.42 & 3.81 \\
\hline TP & 4316 & 0.87 & 0.24 & 521.51 & $-3,0 \mathrm{E}+04$ & 15783,56 \\
\hline DACC $^{*}$ TP & 3422 & 0.75 & 0.05 & 257.99 & $-1.2 \mathrm{E}+04$ & 6711.58 \\
\hline Erro (BTDi) & 3422 & 0.00 & 0.00 & 0.00 & -0.02 & 0.02 \\
\hline
\end{tabular}


TABELA 7 - Modelo de BTD - Book-Tax Differences

\begin{tabular}{l|c|c|c|c}
\hline & Coeficiente & Std. Error & T & Signif.(P) \\
\hline DACC $^{7}$ & .0042962 & .0009553 & 4.50 & $\mathbf{0 , 0 0 0}^{*}$ \\
\hline TP $^{8}$ & $5.92 \mathrm{E}-06$ & $4,34 \mathrm{E}-06$ & 1,60 & 0,111 \\
\hline DACC & & $-1,60$ & 0,110 \\
\hline (Constante) & -.0000186 & .0000116 & -2.47 & 0,014 \\
\hline
\end{tabular}

N. de observações = 2678; Significância do modelo $=0,0000 ; R \cdot, \cdot 70={ }^{2} ;$ RMSE (erro) $=.02013$

*significância a $1 \%{ }^{* *}$ significância a $5 \%$ ***significância a $10 \%$

midade tem sobre o gerenciamento de resultados. Nele, utilizaremos todas as proxys criadas nos modelos anteriores para estudar o efeito da conformidade no gerenciamento de resultado. $\mathrm{Na}$ Tabela 8 , é exibido uma estatística descritiva das variáveis utilizada pelo modelo.

De acordo com o modelo 4, temos que a variável dependente da regressão é a criada através do modelo 3 , obtida por meio do erro gerado pela regressão que será utilizada como medida de gerenciamento de resultado (EM). Neste modelo usamos as duas variáveis de conformidade, conforme no modelo original, gerando resultados explicativos de conformidade dos lucros sob o gerenciamento. Foram usadas também outras variáveis para poder controlar algum efeito aleatório que possa apresentar na regressão. Esses controles são, tamanho (TAM), vendas (VENDAS), alavancagem financeira (ALAV) e retorno sobre os ativos (ROA).
No primeiro momento, faremos a regressão de interesse com a primeira proxy encontrada de conformidade, de acordo com o modelo I (ATWOOD et al. 2010; TANG, 20I5). Nela a variável dependente (gerenciamento de resultados), será explicada (ou não) por uma proxy criada de conformidade, usada como variável independente. Os resultados da regressão indicam que, o modelo estudado é um modelo válido ( $p$ valor $=0,000$ ), que tem um grau de explicação de $92,95 \%$ do gerenciamento de resultado $\left(R^{2}\right.$ ajustado $\left.=\mathbf{0 , 9 2 9 5}\right)$.

Conforme os resultados encontrados, há indicações que o gerenciamento de resultado pode ser afetado pela conformidade. Isso porque as duas variáveis de conformidade explicativas do gerenciamento de resultado, a de BTC e a interação com a adoção do IFRS (BTC_IFRS), são significativas para o modelo estudado. Assim podemos inferir que, a adoção do IFRS tem impacto

TABELA 8 - Modelo de Tang (2015)

\begin{tabular}{l|c|c|c|c|c|c}
\hline Variável & $\mathrm{N}$ & Média & Mediana & Desv. Padrão & Min. & Max. \\
\hline EM & 3503 & 0.26 & 0.21 & 0.37 & -3.42 & 3.81 \\
\hline BTC & 4363 & 0.02 & 0.00 & 0.16 & -2.88 & 2.84 \\
\hline BTDi & 3422 & 0.00 & 0.00 & 0.00 & -0.02 & 0.02 \\
\hline BTC*IFRS & 4363 & 0.00 & 0.00 & 0.06 & -0.91 & 1.94 \\
\hline BTDi*IFRS & 3422 & 0.00 & 0.00 & 0.00 & -0.02 & 0.02 \\
\hline TAM & 4421 & 0.00 & 0.00 & 0.00 & 0.00 & 0.33 \\
\hline VENDAS & 4427 & 0.63 & 0.10 & 2.69 & 0.00 & 54.91 \\
\hline ALAV & 4421 & 0.71 & 0,52 & 4,38 & 0,00 & 316.00 \\
\hline ROA & 4416 & 0,70 & 0,59 & 0,56 & $-0,02$ & 5,84 \\
\hline
\end{tabular}


no gerenciamento de resultados. Ou seja, a conformidade dos lucros, medida conforme o modelo I do estudo, tem efeito sobre o gerenciamento de resultados.

É possível afirmar também, com esse resultado, que, passando para um sistema contábil mais independente, isto é, de um modelo único de demonstrações para um modelo separado (contábil e fiscal), pode se afirmar que haverá aumento no gerenciamento de resultado, em especifico no caso estudado neste trabalho, no comportamento oportunístico dos gerentes de gerenciarem os resultados das demonstrações das empresas. Embora a adoção do IFRS e a conformidade dos lucros tenham efeitos sobre o gerenciamento, as demais variáveis foram significativas para o modelo (variáveis explicativas de controle). Na medida em que o tamanho (TAM) das empresas tem impacto negativo sobre o geren- ciamento, quanto maior a empresa, menor será o nível de gerenciamento de resultados em suas demonstrações. O mesmo é válido para a variável de retorno sobre os ativos (ROA), na qual, quanto maior o retorno menor o nível de gerenciamento. Já as vendas (VENDAS) têm efeito inverso sobre o gerenciamento, quanto maior as vendas, maior será o nível de gerenciamento. A variável alavancagem financeira (ALAV) também é significativa, com efeito positivo sobre o gerenciamento de resultados, quanto maior a alavancagem da empresa, mais a empresa apresentará gerenciamento nas suas demonstrações.

Nesse segundo momento, partiremos para a análise dos efeitos da conformidade sob o gerenciamento de resultados, da proxy de conformidade obtida pelo modelo de regressão 2 do trabalho (TANG, 20I5). Os resultados da regressão indicam que, 0

TABELA 9 - Modelo de ATWOOD et al. (2010)

\begin{tabular}{l|c|c|c|c}
\hline & Coeficiente & Std. Error & $\mathrm{t}$ & Signif.(P) \\
\hline BTC & -1.353992 & .4730795 & -2.86 & $\mathbf{0 , 0 0 0 4 ^ { * }}$ \\
\hline BTC_IFRS & 1.755262 & .472927 & 3.71 & $\mathbf{0 , 0 0 0 ^ { * }}$ \\
\hline TAM & -10.82332 & .4777294 & -22.66 & $\mathbf{0 , 0 0 0 ^ { * }}$ \\
\hline VENDAS & 1.176907 & .0173314 & 67.91 & $\mathbf{0 , 0 0 0 ^ { * }}$ \\
\hline ALAV & .000982 & .0004575 & 2.15 & $\mathbf{0 , 0 3 2}^{* *}$ \\
\hline ROA & -.2912297 & .0034438 & -84.57 & $\mathbf{0 , 0 0 0 ^ { * }}$ \\
\hline (Constante) & .3080631 & .0034314 & 89.78 & 0,000 \\
\hline
\end{tabular}

Núm. de observações = 2695; Significância do modelo =0,000; $R^{2}=0.9295$; RMSE (erro) $=.10132$

*significância a $1 \%$ ** significância a $5 \%$ *** significância a $10 \%$

TABELA 10 - Modelo de TANG (2015)

\begin{tabular}{l|c|c|c|c|}
\hline & Coeficiente & Std. Error & $\mathrm{t}$ & Signif.(P) \\
\hline BTDi & 212.8129 & 14.05897 & 15.14 & $\mathbf{0 , 0 0 0 ^ { * }}$ \\
\hline BTDi_IFRS & -.2339262 & .7121408 & -0.33 & 0,743 \\
\hline TAM & -.9010149 & .6542541 & -1.38 & 0,169 \\
\hline VENDAS & .1039465 & .0726522 & 1.43 & 0,153 \\
\hline ALAV & .0000292 & .000064 & 0.648 & 0,648 \\
\hline ROA & -.0267443 & .0186395 & -1.43 & 0,151 \\
\hline (Constante) & .2213867 & .0070207 & 31.53 & 0,000 \\
\hline
\end{tabular}

Núm. de observações = 2678; Significância do modelo = 0,000; $\mathrm{R}^{2}=0.9932 ; \mathrm{RMSE}$ (erro) = .03129

*significância a $1 \%$ **significância a $5 \%$ *** significância a $10 \%$ 
modelo estudado é um modelo válido ( $\mathbf{p}$ valor $\mathbf{0} \mathbf{0}, \mathbf{0 0 0}$ ), que tem um grau de explicação de $99,32 \%$ do gerenciamento de

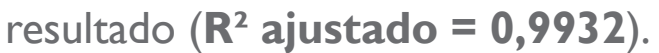

Apresentado os resultados da regressão, verificamos que a única variável com significância para explicação do gerenciamento de resultados é a proxy de conformidade gerada pelo modelo 2 de Tang (20I5). Com significância de $99 \%$, a proxy criada por Tang (20I5) tem impacto positivo sobre o gerenciamento de resultados. Assim, quanto maior o valor da variável BTD menor será a conformidade, temos que, quanto maior $\mathrm{O}$ valor da proxy de conformidade maior, também será o gerenciamento de resultados. Com os resultados podemos observar que, a adoção do IFRS não teve significância sobre o gerenciamento de resultados. Com a adoção do IFRS o esperado seria que ocorresse $o$ aumento da desconformidade, com a adoção de dois modelos de relatórios, assim também aumentando a oportunidade dos gerentes em agir com discricionariedade, por fim gerenciando os resultados das empresas, para mais ou para menos.

\section{CONCLUSÕES}

Neste trabalho, buscamos estudar os efeitos causados pela conformidade do lucro contábil e fiscal no comportamento oportunístico de gerenciamento de resultados na divulgação das demonstrações (contábeis e fiscais) das empresas brasileiras, listadas na B3 - Brasil, Bolsa, Balcão. O estudo se estendeu pelos anos de 1995 a 2016, de onde foram excluídos os anos de 2008 e 2009 , por ser um período de adaptação e adoção (não mandatória) das normas internacionais de contabilidade - IFRS. A conformidade dos lucros é um debate que está em evidência nos estudos interna- cionais e também é um assunto de extrema relevância para o cenário do país. Com a recente adoção das normas internacionais, o país passa de um sistema mais centralizado de informações contábeis para um sistema um pouco mais independente. Várias são as implicações dessa introdução das regras internacionais de demonstração contábeis, que também avançam no campo político, social e econômico. Contudo, não cabe a este trabalho $\circ$ avanço dessa discussão, limitando-se apenas em apontar os efeitos que a conformidade possa ter sobre a qualidade dos números contábeis.

O estudo apresentado se baseou em modelos econométricos utilizados em trabalhos internacionais, que pesquisaram empresas de diversos países (cross-country). Um dos modelos utilizados foi o modelo de Book-Tax Conformity - BTC, desenvolvido no trabalho de Atwood et al. (20l0), que vem sendo empregado em estudos que discutam sobre conformidade dos lucros contábil e fiscal desde então. Um segundo modelo de conformidade foi utilizado para dar consistência a essa proxy, foi o modelo econométrico de Tang (20I5). Esse modelo também utilizado para estudos de empresas em diversos países (cross-country), é uma alternativa ao de Atwood et al. (20l0) para encontrar resultados de conformidade, com foco nas diferenças entre os lucros. Para encontrarmos o gerenciamento de resultados, utilizamos como modelo econométrico uma proxy de accruals discricionários, por meio da regressão proposta por Dechow et al. (1995) - Jones Modificado.

Por fim, esses resultados obtidos das regressões anteriores foram utilizados como variáveis dependente (gerenciamento) e independentes (conformidade), para encontrar o impacto da conformidade sobre 
o gerenciamento de resultados. $O$ modelo do estudo empírico realizado, é um modelo de Tang (20I5), aplicado com algumas adaptações para o propósito deste trabalho.

Os resultados encontrados nas regressões propostas, suportam a hipótese do trabalho de que, existe relação entre a conformidade dos lucros contábil e fiscal com o gerenciamento de resultados. Assim, com base neles, podemos afirmar que o nível de conformidade dos lucros tem influência sobre o gerenciamento de resultados. Isso porque fica implícito que a conformidade dos lucros tem poder de restringir o comportamento oportunístico dos gerentes para gerenciar, para cima ou para baixo, de acordo com seus incentivos, os resultados da empresa. Em última análise, podemos inferir que o nível de conformidade afeta o comportamento oportunístico dos gerentes a fim de gerenciar os resultados das empresas por motivos e incentivos diversos, em que, a qualidade do lucro das empresas será afetada pela conformidade dos lucros.

Em adicional ao resultado, foi encontrado que quanto maior o tamanho da empresa, menor será o nível de gerenciamento de resultados. Também foi encontrado que, de forma positiva, as vendas têm efeito sobre o gerenciamento, de maneira que, quanto maior forem as vendas, maior será o gerenciamento.

Os resultados encontrados são de grande importância, uma vez que abrangem uma pesquisa que se aprofunda no tema de con- formidade dos lucros, a fim de investigar as suas implicações na qualidade dos números contábeis. Utilizando métodos recentes e bem aceitos nos estudos internacionais, a aplicação dessas pesquisas no país, abrangendo um período amplo conseguem confrontar períodos distintos, configurando um trabalho praticamente inédito no país.

Por fim, para pesquisas futuras, indicase aplicar modelos que tenham robustez nos resultados de conformidade. Por uma limitação do trabalho, indica-se também um estudo que possa utilizar dados de empresas que não estejam listadas na B3 (de capital fechado).

Para trabalhos futuros aponta-se, também, o estudo da conformidade do lucro por segmento de atividade empresarial e, ainda, o estudo cross-country, com países de economia similar à brasileira, para efeito de comparação.

Ademais, para trabalhos futuros é recomendada a utilização de novas proxys de conformidade, uma vez que nos testes de robustez foi indicado que a proxy do modelo I (ATOWOOD et al. 2010) não é muito confiável na aplicação e análise de dados das empresas brasileiras. Para pesquisas futuras é indicado também, um estudo mais profundo da qualidade informacional das demonstrações contábeis e a fidedignidade dos seus resultados, indo um pouco mais além do estudado por este trabalho, verificando o impacto direto da conformidade nas informações apresentadas pelas empresas. 
ALI, Ashiq; CHEN, Tai-Yuan; RA- BEATTY, Anne L.; KE, Bin; PETRONI, DHAKRISHNAN, Suresh. Corporate disclosures by family firms. Journal of accounting and economics, v. 44, n. I, p. 238-286, 2007.

ALI, Ashiq; HWANG, Lee-Seok. Country-specific factors related to financial reporting and the value relevance of accounting data. Journal of accounting research, v. 38, n. I, p. I-2I, 2000.

AN, Y.; NAUGHTON, T. The impact of family ownership on firm value and earning quality: Evidence from Korea. In: European Financial Management Association Conference. European Financial Management Association, 2009. p. I-29.

ANDERSON, Ronald C.; MANSI, Sattar A.; REEB, David M. Founding family ownership and the agency cost of debt. Journal of financial economics, v. 68, n. 2, p. 263285, 2003.

ARDISON, Kym Marcel Martins; MARTINEZ, Antonio Lopo; GALDI, Fernando Caio. The effect of leverage on earnings management in Brazil. ASAA-Advances in Scientific and Applied Accounting, v. 5, n. 3, p. 305-324, 2013.

ATWOOD, T. J.; DRAKE, Michael S.; MYERS, Linda A. Book-tax conformity, earnings persistence and the association between earnings and future cash flows. Journal of Accounting and Economics, v. 50, n. I, p. I I I- I 25, 20 I0.

BALL, Ray; SHIVAKUMAR, Lakshmanan. Earnings quality in UK private firms: comparative loss recognition timeliness. Journal of accounting and economics, v. 39 , n. I, p. 83-I 28, 2005.

BASU, Sudipta. The conservatism principle and the asymmetric timeliness of earnings I. Journal of accounting and economics, v. 24, n. I, p. 3-37, 1997.
Kathy R. Earnings management to avoid earnings declines across publicly and privately held banks. The Accounting Review, v. 77, n. 3, p. 547-570, 2002.

BLAYLOCK, Bradley; GAERTNER, Fabio B.; SHEVLIN, Terry. Booktax conformity and capital structure. Review of Accounting Studies, v. 22, n. 2, p. 903-932, 2017.

BLAYLOCK, Bradley; GAERTNER, Fabio; SHEVLIN, Terry. The association between book-tax conformity and earnings management. Review of Accounting Studies, v. 20, n. I, p. |4| - |72, 2015.

BLAYLOCK, Bradley; SHEVLIN, Terry; WILSON, Ryan J. Tax avoidance, large positive temporary book-tax differences, and earnings persistence. The Accounting Review, v. 87, n. I, p. 91-120, 20 II. BURGSTAHLER, David C.; HAIL, Luzi; LEUZ, Christian. The importance of reporting incentives: Earnings management in European private and public firms. The accounting review, v. 81, n. 5, p. 983-1016, 2006.

BURGSTAHLER, David; DICHEV, Ilia. Earnings management to avoid earnings decreases and losses. Journal of accounting and economics, v. 24, n. I, p. 99-I26, 1997. CHAN, K. Hung; LIN, Kenny Z.; TANG, Feng. Tax effects of booktax conformity, financial reporting incentives, and firm size. Journal of International Accounting Research, v. I2, n. 2, p. I-25, 20 I3. COELHO, Antonio Carlos; GALDI, Fernando Caio; LOPES, Alexsandro Broedel. Determinants of asymmetric loss recognition timeliness in public and private fir$\mathrm{ms}$ in Brazil. Emerging Markets Review, v. 31, p. 65-79, 2017.
COELHO,Antonio Carlos; LIMA, Iran Siqueira. Qualidade informacional e conservadorismo nos resultados contábeis publicados no Brasil. Revista Contabilidade \& Finanças, v. 18, n. 45, p. 38-49, 2007.

DECHOW, Patricia M.; DICHEV, Ilia $D$. The quality of accruals and earnings: The role of accrual estimation errors. The accounting review, v. 77, n. s-I, p. 35-59, 2002.

DECHOW, Patricia M.; KOTHARI, Sagar P.; WATTS, Ross L. The relation between earnings and cash flows. Journal of accounting and Economics, v. 25, n. 2, p. |33-168, 1998.

DECHOW, Patricia M.; SLOAN, Richard G.; SWEENEY, Amy P. Causes and consequences of earnings manipulation: An analysis of firms subject to enforcement actions by the SEC. Contemporary accounting research, v. I3, n. I, p. I-36, 1996.

DECHOW, Patricia; GE, Weili; SCHRAND, Catherine. Understanding earnings quality: $A$ review of the proxies, their determinants and their consequences. Journal of Accounting and Economics, v. 50, n. 2, p. 344-40I, 2010.

DESAI, Mihir A. The degradation of reported corporate profits. Journal of Economic Perspectives, v. 19, n. 4, p. |7|-192, 2005.

DESAI, Mihir A.; DHARMAPALA, Dhammika. Earnings management, corporate tax shelters, and booktax alignment. National Tax Journal, p. I69-I86, 2009.

FORMIGONI, Henrique; ANTUNES, Maria Thereza Pompa; PAULO, Edilson. Diferença entre o lucro contábil e lucro tributável: uma análise sobre o gerenciamento de resultados contábeis e gerenciamento tributário nas companhias 
abertas brasileiras. BBR Brazilian Business Review, v. 6, n. I, p. 44-6I, 2009.

GIVOLY, Dan; HAYN, Carla K.; KATZ, Sharon P. Does public ownership of equity improve earnings quality? The Accounting Review, v. 85, n. I, p. 195-225, 2010.

GOMES MACHADO, Débora; BEU-

REN, Ilse Maria. Gerenciamento de resultados: análise das publicações em periódicos brasileiros de contabilidade. Enfoque: $\mathbf{R e}$ flexão Contábil, v. 33, n. I, $20 \mid 4$. HANLON, Michelle. The persistence and pricing of earnings, accruals, and cash flows when firms have large book-tax differences. The accounting review, v. 80, n. I, p. I37-166, 2005.

HANLON, Michelle; MAYDEW, Edward L.; SHEVLIN, Terry J. Book-tax conformity and the information content of earnings. 2006.

HANLON, Michelle; MAYDEW, Edward L.; SHEVLIN, Terry. An unintended consequence of booktax conformity: A loss of earnings informativeness. Journal of Accounting and Economics, v. 46, n. 2-3, p. 294-3। I, 2008.

HEALY, Paul M.; WAHLEN, James M. A review of the earnings management literature and its implications for standard setting. Accounting horizons, v. I3, n. 4, p. 365-383, 1999.

HUNG, Mingyi. Accounting standards and value relevance of financial statements: An international analysis. Journal of accounting and economics, v. 30 , n. 3 , p. 40I-420, 2000.

KOTHARI, Sagar P.; LEONE, Andrew J.;WASLEY, Charles E. Performance matched discretionary accrual measures. Journal of accounting and economics, v. 39, n. I, p. 163-197, 2005.
LIN, Kenny Z.; MILLS, Lillian F.; ZHANG, Fang. Public versus private firm responses to the tax rate reduction in China. The Journal of the American Taxation Association, v. 36, n. I, p. |37-|63, 2013.

LOPES, Alexsandro Broedel; MARTINS, Eliseu. Teoria da contabilidade: uma nova abordagem. Atlas, 2007.

LOPO MARTINEZ, Antonio. Detectando earnings management no Brasil: estimando os accruals discricionários. Revista Contabilidade \& Finanças-USP, v. 19, n. 46, 2008.

LOPO MARTINEZ, Antonio. Gerenciamento de resultados no Brasil: um survey da literatura. BBR -Brazilian Business Review, v. I0, n. 4, 2013.

MACHADO, Débora; BENETTI, Juliana; BEZERRA, Francisco. Análise da produção científica sobre earnings management em periódicos brasileiros e internacionais de contabilidade. Revista de Gestão dos Países de Língua Portuguesa, v. 10, n. 4, p. 50-66, 201 I.

MILLS, Lillian F. Book-tax differences and Internal Revenue Service adjustments. Journal of Accounting research, v. 36, n. 2 , p. 343356, 1998.

MILLS, Lillian F;; NEWBERRY, Kaye J.The influence of tax and nontax costs on book-tax reporting differences: Public and private firms. Journal of the American Taxation Association, v. 23, n. I, p. I-19, 200।.

RAMOS FERREIRA, Felipe et al. Book-tax differences e gerenciamento de resultados no mercado de ações do Brasil. RAE-Revista de Administração de Empresas, v. 52, n. 5, 2012.

SUNDER, Shyam; CYERT, Richard $M$. Theory of accounting and control. South-Western College
Pub., 1997.

SUNDVIK, Dennis. Book-tax conformity and earnings management in response to tax rate cuts. Journal of International Accounting, Auditing and Taxation, v. 28, p. $3 \mid-42,2017$.

TANG, Tanya YH. Book-tax differences: a function of accounting-tax misalignment, earnings management and tax management: empirical evidence from China. In: American Accounting Association Annual Meeting, Washington DC. 2006.

TANG, Tanya YH. Does book-tax conformity deter opportunistic book and tax reporting? An international analysis. European Accounting Review, v. 24, n. 3 , p. 44I-469, 20 I 5.

WANG, Dechun. Founding family ownership and earnings quality. Journal of accounting research, v. 44, n. 3, p. 619-656, 2006.

WATRIN, Christoph; EBERT, Nadine; THOMSEN, Martin. Book-tax conformity and earnings management: insights from European one-and two-book systems. The Journal of the American Taxation Association, v. 36, n. 2, p. 55-89, 2014.

WATRIN, Christoph; POTT, Christiane; ULLMANN, Robert. The effects of book-tax conformity and tax accounting incentives on financial accounting: evidence from public and private limited companies in Germany. International Journal of Accounting, Auditing and Performance Evaluation, v. 8, n. 3, p. 274-302, 2012.

WHITAKER, Celia. Bridging the booktax accounting gap. The Yale Law Journal, p. 680-726, 2005.

YIN, George K. Getting serious about corporate tax shelters: Taking a lesson from history. SMUL Rev., v. 54 , p. 209,200 I. 